\title{
fNIRS-based brain-computer interfaces: a review
}

\author{
Noman Naseer ${ }^{1}$ and Keum-Shik Hong ${ }^{1,2 *}$ \\ ${ }^{1}$ Department of Cogno-Mechatronics Engineering, Pusan National University, Busan, Republic of Korea \\ ${ }^{2}$ School of Mechanical Engineering, Pusan National University, Busan, Republic of Korea
}

\section{Edited by:}

Arthur M. Jacobs, Freie Universität Berlin, Germany

\section{Reviewed by:}

Martin J. Herrmann, Universtity of Würzburg, Germany

Vadim Nikulin, Charite University

Hospital, Germany

\section{*Correspondence}

Keum-Shik Hong, Department of

Cogno-Mechatronics Engineering,

Pusan National University,

2 Busandaehak-ro, Guemjeong-gu,

Busan 609-735, Republic of Korea

e-mail: kshong@pusan.ac.kr
A brain-computer interface $(\mathrm{BCl})$ is a communication system that allows the use of brain activity to control computers or other external devices. It can, by bypassing the peripheral nervous system, provide a means of communication for people suffering from severe motor disabilities or in a persistent vegetative state. In this paper, brain-signal generation tasks, noise removal methods, feature extraction/selection schemes, and classification techniques for fNIRS-based $\mathrm{BCl}$ are reviewed. The most common brain areas for fNIRS $\mathrm{BCl}$ are the primary motor cortex and the prefrontal cortex. In relation to the motor cortex, motor imagery tasks were preferred to motor execution tasks since possible proprioceptive feedback could be avoided. In relation to the prefrontal cortex, fNIRS showed a significant advantage due to no hair in detecting the cognitive tasks like mental arithmetic, music imagery, emotion induction, etc. In removing physiological noise in fNIRS data, band-pass filtering was mostly used. However, more advanced techniques like adaptive filtering, independent component analysis (ICA), multi optodes arrangement, etc. are being pursued to overcome the problem that a band-pass filter cannot be used when both brain and physiological signals occur within a close band. In extracting features related to the desired brain signal, the mean, variance, peak value, slope, skewness, and kurtosis of the noised-removed hemodynamic response were used. For classification, the linear discriminant analysis method provided simple but good performance among others: support vector machine (SVM), hidden Markov model (HMM), artificial neural network, etc. fNIRS will be more widely used to monitor the occurrence of neuro-plasticity after neuro-rehabilitation and neuro-stimulation. Technical breakthroughs in the future are expected via bundled-type probes, hybrid EEG-fNIRS BCl, and through the detection of initial dips.

Keywords: brain-computer interface, functional near-infrared spectroscopy (fNIRS), feature extraction, feature classification, physiological noise, brain-machine interface

\section{INTRODUCTION}

A brain-computer interface (BCI) system provides its users with control channels that are independent of the brain's output channels (i.e., the peripheral nervous system and muscles) (Wolpaw et al., 2002). Such systems can be used as a means for communications and restoration of motor functions (through a neuroprosthesis) for people with motor disorders such as amyotrophic lateral sclerosis (ALS) and spinal cord injury, and/or people in the persistent locked-in state (LIS). It can also be used as a neurorehabilitation tool to improve motor and/or cognitive performance of such people.

A typical BCI system consists of five stages (see Figure 1): brain-signal acquisition, preprocessing, feature extraction/selection, classification, and application interface. In the first brain-signal acquisition stage, suitable signals are acquired using an appropriate brain-imaging modality. Since the acquired signals are normally weak and contain noises (physiological and instrumental) and artifacts, preprocessing is needed, which is the second stage. In the third stage, some useful data so called "features" are extracted. These features, in the fourth stage, are classified using a suitable classifier. Finally, in the fifth stage, the classified signals are transmitted to a computer or other external devices for generating the desired control commands to the devices. In neurofeedback applications, a real-time display of brain activity is desirable, which enables self-regulation of brain functions. Figure 1 depicts a schematic of (hybrid) functional near-infrared spectroscopy (fNIRS) and electroencephalography (EEG) BCI.

Several modalities have been used for brain signal acquisition, which include EEG (Wolpaw et al., 2002; Turnip et al., 2011; Turnip and Hong, 2012; Wang et al., 2012; Hwang et al., 2013; Kleih and Kubler, 2013; Ko and Sim, 2013; Hammer et al., 2014; Kim et al., 2014; Soekadar et al., 2014), magnetoencephalography (MEG) (Mellinger et al., 2007; Buch et al., 2008; Sardouie and Shamsollahi, 2012), functional magnetic resonance imaging (fMRI) (Weiskopf et al., 2004; LaConte, 2011; van der Heiden et al., 2014), and fNIRS (Ferrari et al., 1985, 2004; Kato et al., 1993; Hu et al., 2013; Bhutta et al., 2014; Rea et al., 2014; Santosa et al., 2014). Among them, fNIRS is relatively new, which uses near-infrared-range light (usually of $650 \sim 1000 \mathrm{~nm}$ wavelength) to measure the concentration changes of oxygenated hemoglobin $(\mathrm{HbO})$ and deoxygenated hemoglobin (HbR) (Villringer et al., 


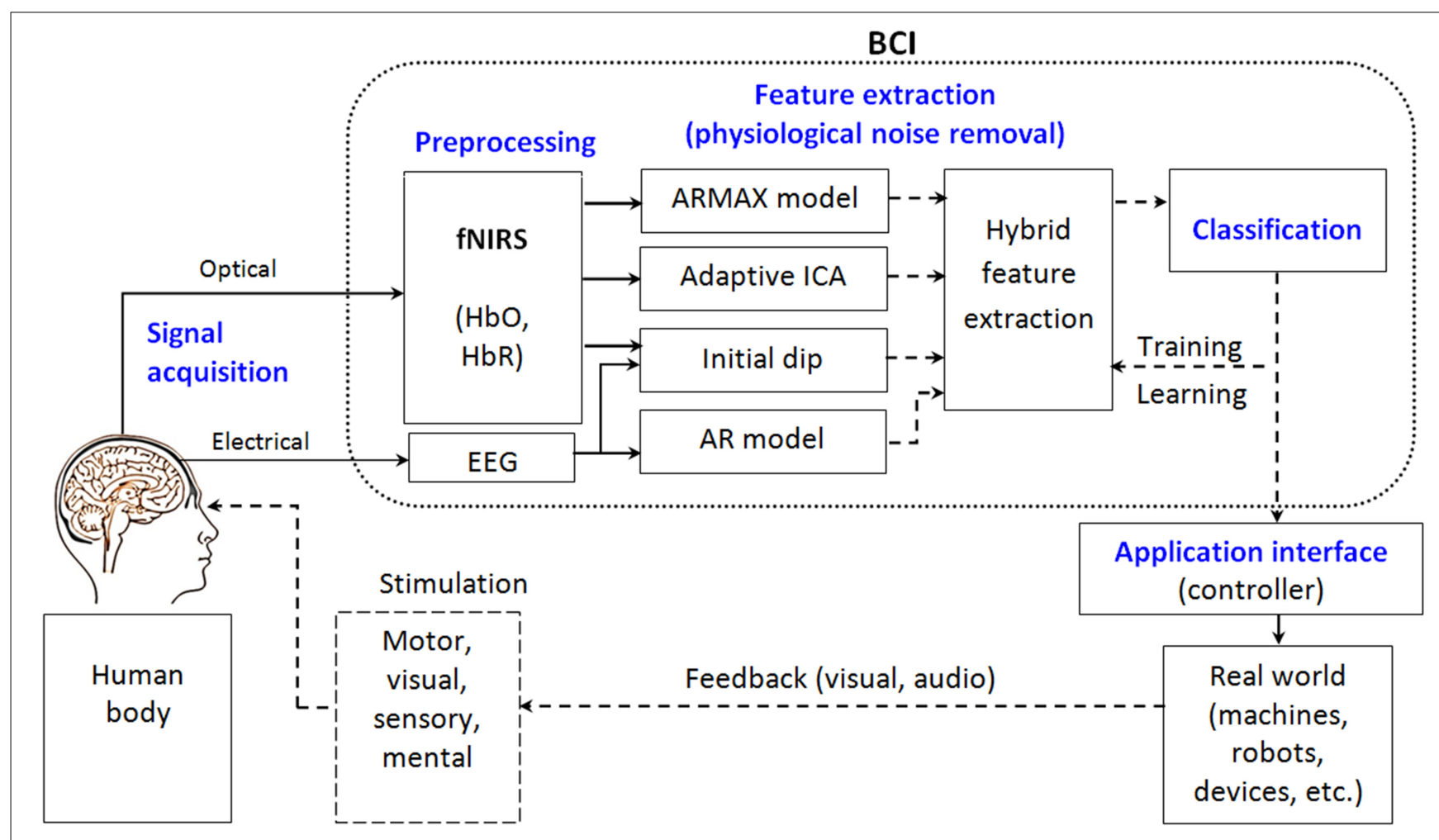

(AR: auto-regressive, ARMAX: auto-regressive moving average with exogenous signals, ICA: independent component analysis)

FIGURE 1 | Schematic of a hybrid fNIRS-EEG BCI.

1993; Hoshi et al., 1994; Hoshi and Tamura, 1997; Villringer and Chance, 1997; Boas et al., 2004a,b; Hong and Nguyen, 2014). Its main advantages are relatively low cost, portability, safety, low noise (compared to fMRI), and easiness to use. Unlike EEG and MEG, its data are not much susceptible to electrical noise, since it is an optical imaging modality. fNIRS measures the blood flow changes in the local capillary network caused by neuron firings. Since the hemoglobin is an oxygen carrier, the changes of $\mathrm{HbO}$ and $\mathrm{HbR}$ concentration levels after a neuronal activation can be related to the relevant neuronal firings. ANIRS uses near-infrared (NI) light emitter-detector pairs operating with two or more wavelengths. The NI light emitted into the scalp diffuses through the brain tissues resulting in multiple scattering of photons. Some of these photons exit the head after passing through the cortical region of the brain, wherein the chromophores (i.e., $\mathrm{HbO}$ and $\mathrm{HbR}$ ) are changing in time. These exited photons are then detected by using strategically positioned detectors. Since $\mathrm{HbO}$ and $\mathrm{HbR}$ have different absorption coefficients for different wavelengths of NI light, the relationship between the exiting-photon intensity and the incident-photon intensity can be used to calculate the changes of the concentrations of $\mathrm{HbO}$ and $\mathrm{HbR}\left[\Delta c_{\mathrm{HbO}}(t)\right.$ and $\left.\Delta c_{\mathrm{HbR}}(t)\right]$ along the path of the photons by applying the modified Beer-Lamberts law (Delpy et al., 1988).
The principle of fNIRS measurement, first reported by Jobsis (1977), has been applied to the study of cerebral hemodynamics for more than two decades, even though its BCI use is only a few years old. The first study who demonstrated the feasibility of fNIRS for BCI was Coyle et al. (2004). They asked the subjects to perform motor imagery of continuous squeezing and releasing of a soft ball. Based on the activity threshold of $\Delta c_{\mathrm{HbO}}(t)$, they determined whether the brain was activated or at rest.

In 2007, three studies demonstrated the feasibility of controlling the output of fNIRS BCI: Coyle et al. (2007) used a custom-built fNIRS system (named Mindswitch) to test on-off control. Their protocol consisted of two options alternately presented to the subjects: When a desired option was highlighted, the subject performed motor imagery of squeezing and releasing a soft ball to enhance the HbO signals in the motor cortex and, in this way, expressed their choice mentally. The signals during motor imagery were classified against those during the rest period with an average accuracy of more than $80 \%$. Sitaram et al. (2007) showed that fNIRS signal patterns during execution movement and imagery were distinguishable with the accuracy of $80 \%$ (or above) using support vector machines (SVM) and hidden Markov model (HMM). On the other hand, the first investigation on ALS patients was done by Naito et al. (2007): Forty ALS patients (17 of them were totally locked-in) were asked to encode their response 
to several questions as "yes" or "no." They were requested to respond "yes" by performing mental calculation, music imagery and other such tasks, and to respond "no" by remaining relaxed. The instantaneous amplitude and phase of the light-intensity signals were then used as the features for a quadratic discriminant analysis classifier, which successfully decoded the responses of $70 \%$ of the ALS patients who were not totally locked-in. However, for totally locked-in ALS patients, the method worked only $40 \%$ of subjects (with the classification accuracy of about $80 \%$ ).

In 2008, Utsugi et al. (2008) showed the feasibly of a "Go-Stop" control. They measured the spatiotemporal averages of $\Delta c_{\mathrm{HbO}}(t)$ and $\Delta c_{\mathrm{HbR}}(t)$ arising from mental calculations. Bauernfeind et al. (2008) developed an fNIRS system and reported that changes in $\Delta c_{\mathrm{HbO}}(t)$ and $\Delta c_{\mathrm{HbR}}(t)$ were observed during mental arithmetic tasks over the prefrontal cortex. The measured signals were relatively stable across 13 subjects. Based on that, the authors suggested its application to BCI.

In 2009, Luu and Chau (2009) demonstrated the preferencedecoding possibilities using fNIRS signals acquired from the prefrontal cortex. Nine subjects were asked to mentally evaluate two presented drinks and decide which one they preferred. Instead of using a specific activity to choose the preferred drink, they used the direct neural correlates in decision making. The accuracy of this preference decoding, using light-intensity signals directly and linear discriminant analysis (LDA), was around $80 \%$. In the same year, Tai and Chau (2009) showed the feasibility for BCI development of fNIRS-signal classification from emotion-induction tasks. The subjects preformed several trials of positive- and negative-emotion-induction tasks, and the optimal features were selected using a genetic algorithm. Then, LDA and SVM were used to classify different sets of features to the average accuracies ranging from 75 to $94 \%$. Since 2009 , several studies have successfully demonstrated the use of fNIRS for efficient BCI. Although EEG-based BCIs are most common noninvasive versions, the trend of using fNIRS for BCI is continuously increasing.

\section{BRAIN-SIGNAL ACOUISITION}

BCI uses brain signals to collect information on the user's intensions. The first step in developing an fNIRS-BCI system is to acquire suitable brain signals. The two most common brain areas are the primary motor cortex and the prefrontal cortex. Signals corresponding to motor execution and motor imagery tasks are acquired from the motor cortex; whereas those corresponding to mental arithmetic, mental counting, music imagery, landscape imagery, etc. are acquired from the prefrontal cortex. Although several different emitter-detector configurations have been used in these two areas, the emitter-detector distance is usually kept within a specific range, as it plays an important role in fNIRS measurement. For example, an increase in emitter-detector distance corresponds to an increase in imaging depth (McCormick et al., 1992). To measure hemodynamic response signals from the cortical areas, an emitter-detector separation of around $3 \mathrm{~cm}$ was suggested (Gagnon et al., 2012). A separation of less than $1 \mathrm{~cm}$ might contain only skin-layer contribution, whereas that of more than $5 \mathrm{~cm}$ might result in weak and therefore unusable signals (Gratton et al., 2006). A typical emitter-detector configuration on the head and the paths traveled by light to reach two detectors are shown in Figure 2. A suitable number of emitter/detector pairs for adequate extraction of neuronal activity vary depending on the type of brain signals that are used for BCI purpose. For the prefrontal cortex, 3 emitters and 8 detectors may be enough to adequately acquire most brain signals corresponding to prefrontal tasks (Luu and Chau, 2009; Power et al., 2010, 2011, 2012a,b; Khan et al., 2014; Naseer et al., 2014). For brain activities corresponding to motor cortex tasks, 6 emitters and 6 detectors can cover the entire motor cortex. In the previous studies, 4 emitters and 4 detectors (Sitaram et al., 2007), 6 emitters and 6 detectors (Naseer and Hong, 2013), and 5 emitters and 4 detectors have been applied to acquire motor-cortex activities.

\section{MOTOR CORTEX ACTIVITIES}

Activities from the primary motor cortex are a good choice for fNIRS-BCI application, as they are natural means of providing BCI control over external devices. Moreover, these might also be useful from the perspective of neurorehabilitation. The two most commonly acquired activities from the motor cortex are motor execution and motor imagery.

\section{Motor execution}

The motor execution task stands for moving a body part to activate the motor cortex, which involves the development of muscular tensions through muscular actions. Since motor execution involves contraction of muscles, motor execution-based BCIs are affected by proprioceptive feedback from contracting muscles and, therefore, the neuronal modulation may not be solely from the central nervous system. Several motor execution tasks including finger tapping (Cui et al., 2010a,b; Seo et al., 2012), hand tapping (Hai et al., 2013; Khan et al., 2014), arm lifting (Shin and

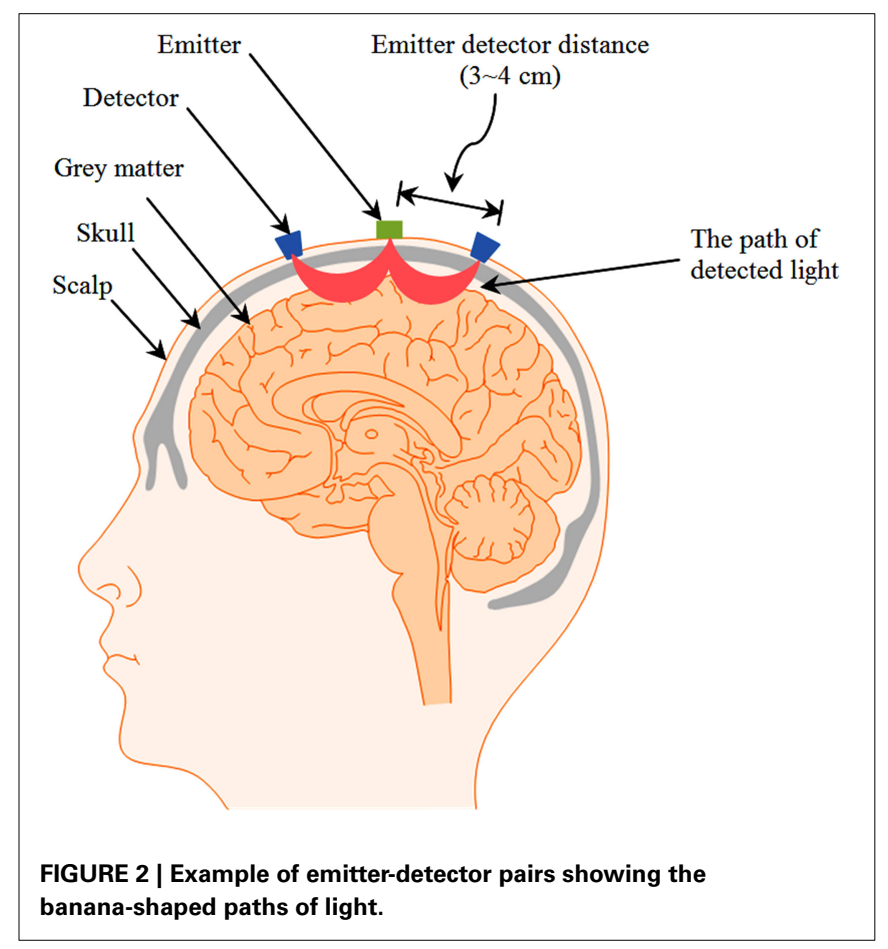


Jeong, 2014), knee extension (Shin and Jeong, 2014) and hand grasping/gripping (Nagaoka et al., 2010; Fazli et al., 2012) have been used in the previous studies.

\section{Motor imagery}

Motor imagery can be defined as a covert cognitive process of kinesthetic imagining of the movement of one's own body part without the involvement of muscular tension, contraction or flexion. Since the primary objective of BCI is to form a communication pathway for motor-disabled people, motor imagery is one of the most commonly utilized tasks in fNIRS-BCI. The motor imagery tasks include imagination of the squeezing of a soft ball (Coyle et al., 2004, 2007; Stangl et al., 2013), covert imagery of a simple or complex sequence of finger tapping (Sitaram et al., 2007; Holper and Wolf, 2011), imagination of feet tapping (Kaiser et al., 2014), imagination of hand grasping/gripping (Nagaoka et al., 2010; Fazli et al., 2012; Kaiser et al., 2014), imagination of wrist flexion (Naseer and Hong, 2013), imagination of flexion and extension of elbow (Mihara et al., 2013), and folding and unfolding of specific fingers (Mihara et al., 2013). Unlike motor execution tasks, the motor imagery signals are free of proprioceptive feedback.

\section{PREFRONTAL CORTEX ACTIVITIES}

The activities in the prefrontal cortex are also a good choice for fNIRS-BCI, because they involve less motion artifacts and signal attenuation due to the slippage in hairs. Also, they are likely to be more effective in the case of motor-function related disability. Given these advantages, most studies have used the prefrontal activities showing promising results (Naito et al., 2007; Bauernfeind et al., 2008, 2011; Utsugi et al., 2008; Luu and Chau, 2009; Power et al., 2010, 2011, 2012a,b; Abibullaev et al., 2011; Falk et al., 2011; Tanaka and Katura, 2011; Abibullaev and An, 2012; Adhika et al., 2012; Chan et al., 2012; Hu et al., 2012; Moghimi et al., 2012; Sagara and Kido, 2012; Faress and Chau, 2013; Power and Chau, 2013; Stangl et al., 2013; Hwang et al., 2014; Naseer et al., 2014; Schudlo and Chau, 2014; Hong et al., 2015). Some of the commonly used prefrontal activities for fNIRS-BCI are mental arithmetic, music imagery, mental counting, and landscape imagery.

\section{Mental arithmetic}

Mental arithmetic (sometimes called mental calculation) means performing covert calculation using the brain without any help in the form of paper, pen, calculator, computer, etc. It activates the prefrontal cortex. Since it does not involve any body movement, it is widely used for fNIRS-BCI. A number of studies have successfully demonstrated its feasibility as a mental task for BCI (Naito et al., 2007; Bauernfeind et al., 2008, 2011; Utsugi et al., 2008; Power et al., 2010, 2011, 2012a,b; Adhika et al., 2012; Sagara and Kido, 2012; Power and Chau, 2013; Stangl et al., 2013; Hwang et al., 2014; Naseer et al., 2014; Hong et al., 2015). Mental arithmetic entails mental multiplication (Hwang et al., 2014) or other arithmetic tasks. However, the most commonly utilized mental arithmetic is backwards subtraction, which involves subtraction of a small number (for example, a two-digit number) from a large number (for example, a three-digit number) with successive subtraction of a randomly appearing small number from the result of the previous subtraction (e.g., 450-15, 435-10, 425-19, etc.) (Power et al., 2010; Hwang et al., 2014; Naseer et al., 2014).

\section{Music imagery}

Music imagery (also called mental singing) consists of organizing and analyzing music in the brain without any external auditory stimulus. Naito et al. (2007), Power et al. (2010), Falk et al. (2011), Power et al. (2011), Chan et al. (2012) and Hwang et al. (2014) successfully demonstrated music imagery as a brain activity that can be effectively used for fNIRS-BCI.

\section{Other prefrontal activities}

Besides mental arithmetic and music imagery, various other tasks in the prefrontal cortex have been shown to work well. These include mental counting (Naito et al., 2007; Khan et al., 2014), landscape imagery (Naito et al., 2007), mental character writing (Hwang et al., 2014), object rotation (Abibullaev et al., 2011; Abibullaev and An, 2012; Faress and Chau, 2013; Hwang et al., 2014), change-detection tasks (Tanaka and Katura, 2011), labyrinth tasks (Misawa et al., 2012), and emotion-induction tasks (Tai and Chau, 2009; Moghimi et al., 2012). Some studies have demonstrated direct decoding of neural correlates corresponding to subjective preferences (Luu and Chau, 2009), deception (Hu et al., 2012), visual stimuli (Faress and Chau, 2013), and others (Ayaz et al., 2009, 2012).

The best selection of optimal mental activities for the improvement of classification accuracy remains an open question. Hwang et al. (2014) evaluated the use of a variety of mental task combinations for BCI. These tasks included motor imagery (right- and left-hand imagery and foot imagery), mental singing, mental arithmetic (multiplication and subtraction), mental rotation, and mental character writing. Out of the 28 different combinations tested, the mental arithmetic/mental rotation and mental arithmetic/right-hand motor imagery combinations yielded the best LDA classification results using mean hemoglobin concentration values. Prefrontal activities have been used in more than half of fNIRS-BCI studies, owing primarily to the easy application of ANIRS to the prefrontal area. Activity selection, however, depends on the given fNIRS-BCI application. For example, for the purposes of limb neurorehabilitation, it is desirable to use motor cortex activities.

\section{PREPROCESSING}

The acquired ANIRS signals can contain various noises, which can be categorized into instrumental noise, experimental error, and physiological noise. Since the instrumental noise and experimental error are not related to the brain activities, it is better to remove them prior to converting the raw optical density signals to the concentration changes of $\mathrm{HbO}$ and $\mathrm{HbR}$ through the modified Beer-Lambert law (Huppert et al., 2009).

\section{REMOVAL OF INSTRUMENTAL NOISE}

Instrumental noise is the noise of fNIRS signals present in hardware or caused by the surrounding environment (i.e., instrumental degradation is an example). It usually involves (constant) high frequencies. Such high frequency can be easily removed by a low-pass filter (for instance, $3 \sim 5 \mathrm{~Hz}$ of cutoff frequency). 
Furthermore, by minimizing the variation of the external light, instrument noise can be significantly reduced.

\section{REMOVAL OF EXPERIMENTAL ERRORS}

Experimental errors include motion artifacts like head motions, which causes the movement of optodes from the assigned positions. This can cause a sudden change in the light intensity resulting in a spike-like noise. Several methods for motion-artifact correction have been proposed in the literature; the Wiener filtering-based method (Izzetoglu et al., 2005), eigenvector-based spatial filtering (i.e., principle component analysis (PCA)-based filtering) (Zhang et al., 2005), wavelet-analysis-based methods (Sato et al., 2006; Power et al., 2010), Savitzky-Golay type filters (Hai et al., 2013; Shin and Jeong, 2014), and others (Cui et al., 2010a,b; Fekete et al., 2011; Cooper et al., 2012). Please see Cooper et al. (2012) for thorough comparison of various techniques.

\section{PHYSIOLOGICAL NOISE}

Physiological noises include those due to heartbeat $(1 \sim 1.5 \mathrm{~Hz})$, respiration $(0.2 \sim 0.5 \mathrm{~Hz})$, Mayer waves $(\sim 0.1 \mathrm{~Hz})$, which are related to blood pressure fluctuations (Boas et al., 2004a,b; Zhang et al., 2005; Franceschini et al., 2006; Huppert et al., 2009). Several methods including band-pass filtering, adaptive filtering, PCA, and independent component analysis (ICA) have been used to remove them.

\section{BAND-PASS FILTERING}

Since the frequency ranges of aforementioned physiological signals are usually known, a band-pass filter can be an effective means. Some fNIRS-BCI studies have shown promising results using a simple low-pass, or a high-pass, or a band-pass filtering to remove physiological noises (Coyle et al., 2004, 2007; Naito et al., 2007; Sitaram et al., 2007; Bauernfeind et al., 2008; Luu and Chau, 2009; Power et al., 2010, 2011; Hu et al., 2012; Liu et al., 2013; Hong et al., 2015).

Various cut-off frequencies for band-pass filtering have been reported in the literature: For example, Luu and Chau (2009), Power et al. (2011), Hu et al. (2012) and Tomita et al. (2014) have used the frequency bands of $0.01 \sim 0.8 \mathrm{~Hz}, 0.1 \sim 0.5 \mathrm{~Hz}$, $0.01 \sim 0.2 \mathrm{~Hz}$, and $0.1 \sim 0.5 \mathrm{~Hz}$, respectively. In general, the band of $0.1 \sim 0.4 \mathrm{~Hz}$ can effectively remove a large portion of physiological noises including heartbeat and Mayer waves without eliminating the fNIRS signal elicited by a task of $10 \mathrm{~s}$ period. The types of band-pass filtering include Butterworth filters (Luu and Chau, 2009; Naseer and Hong, 2013; Naseer et al., 2014), elliptic filters (Hu et al., 2012), and Chebyshev filters (Sitaram et al., 2007; Power et al., 2012b). However, no absolute advantage of a particular filtering method over others has been reported yet.

\section{ADVANCED FILTERING METHODS}

Band-pass filtering cannot be used to filter physiological noises whose frequencies overlap with the band of the hemodynamic response signal, for example, due to respiration. Therefore, other methods, such as adaptive filtering (Zhang et al., 2007; Hu et al., 2010; Aqil et al., 2012a,b; Kamran and Hong, 2013, 2014), PCA (Zhang et al., 2005), and ICA (Kohno et al., 2007; Santosa et al., 2013), have also been used to remove physiological noise. To account for physiological noises, additional noise-related elements can be added into the regression model. In addition to modeling the canonical functional response, a series with adaptive amplitudes and phase components in order to model specific physiological noise contribution from heartbeat, respiration, and blood pressure can be included. The auto-regressive moving average with exogenous signals (ARMAX) model-based approach incorporating physiological signals as exogenous signals can be used to predict the brain state during a particular cognitive task. The fNIRS signal at each channel can be regarded as an output from a linear combination of various components. The components include the dynamical characteristics of the $\mathrm{HbO}$ and HbR changes in a specific brain region (the influence from the current/previous stimuli), the physiological signals, the baseline fluctuation, and other noises.

\section{ICA AND PCA}

ICA can separate physiological noises from the mixed signals allowing the restoration of the original hemodynamic signals. The independent components (ICs) associated with the physiological signals can be identified by their spectral densities. Isolating the main IC associated with the original hemodynamic response results in a physiological-noise-free signal. Hu et al. (2011) and Santosa et al. (2013) used ICA to separate physiological noise from the original signals. Then, the original hemodynamic response was reconstructed using all the ICs (with weights derived from their $t$-values) as well as the primary IC. They applied the proposed method to a mental arithmetic task and compared the results with those of the conventional low-pass filtering method, revealing that the ICA method outperformed the low-pass filtering method. Funane et al. (2014) used ICA to evaluate signal contributions from the shallow and deep tissue layers using multi-distance optodes. They assumed that the optical path length in the shallow layer did not change, but it increased linearly with the increase of emitter-detector distance. The reconstructions of the deep and shallow layer signals were performed by summing all the ICs that had been weighted by the deep and shallow contribution ratio in accordance with the emitter-detector distance.

PCA can be used to remove physiological noises (similarly to the case of motion-artifact removal), because systematic fluctuations are covariant among fNIRS measurements from different channels. Reducing such covariance, accordingly, filters systematic physiological noises from the signals. However, the performance of PCA is greatly dependent on the number of channels and the number of eigenvectors to be removed (Cooper et al., 2012) and, therefore, PCA is not suggested for physiological noise removal when the number of channels is small. Furthermore, a real-time application of ICA for physiological noise removal is still under investigation (a moving window approach for computing ICs can be explored). Henceforth, due to the non-realtimeness of the ICA approach, band-pass filtering techniques are still dominant (Mihara et al., 2012, 2013; Kober et al., 2014).

The fNIRS signals are also affected by the skin blood flow and other contributions from the superficial tissues (Kohno et al., 2007; Takahashi et al., 2011; Kirilina et al., 2012, 2013; Sato 
et al., 2013). It has been shown that the removal of these artifacts from cerebral signals is possible by employing several different methods: the use of additional short-distance detector(s) (Saager and Berger, 2005; Luu and Chau, 2009; Saager et al., 2011), adaptive filtering (Zhang et al., 2009), statistical parametric mapping (SPM) in which the artifacts are included as regressors into the model (Tachtsidis et al., 2010), and ICA (Kohno et al., 2007; Funane et al., 2014). Kohno et al. (2007) revealed that the spatial distribution of one of the ICs was directly related to the skin blood flow, which was again verified by a laser Doppler tissue blood flow meter. Funane et al. (2014), on the other hand, used ICA to separate the absorption changes in deep and shallow tissues (due to the scalp and the skin) using multiple emitterdetector distances. Zhang et al. (2007, 2009) used an adaptive filter to estimate the global interference in the signals measured from short emitter-detector separations. This global interference was then removed from the target signals measured from long emitter detector separations.

\section{FEATURE EXTRACTION/SELECTION}

After data preprocessing, the different brain activities are classified on the basis of certain features. In fNIRS-BCI, although some features are extracted directly from detected light-intensity signals (Naito et al., 2007; Luu and Chau, 2009; Power et al., 2010, 2011), most are extracted from hemodynamic signals. The reason for this is that $\mathrm{HbO}, \mathrm{HbR}$, total hemoglobin ( $\mathrm{HbT})$, and cerebral oxygen exchange $(\mathrm{COE}=\mathrm{HbO}-\mathrm{HbR})$ provide more options for selection of appropriate features. Selection of an optimal feature set for classification is essential for good classification. It is necessary to select such features that have similarities with a certain class and differences from other classes. Different combinations of such features provide the necessary discriminatory information for classification.

\section{HEURISTIC METHODS}

After noise removal, the shape of the hemodynamic signal is usually clear. By observing the hemodynamic signals arising from different activities, one can determine the differences in the signals: peak amplitude, mean value, variance, slope, skewness, kurtosis, etc. These can then be used as features for classification of different signals. The most commonly used features for discrimination of different activities for fNIRS-BCI are signal mean (Coyle et al., 2004, 2007; Sitaram et al., 2007; Luu and Chau, 2009; Power et al., 2010; Holper and Wolf, 2011; Fazli et al., 2012; Moghimi et al., 2012; Faress and Chau, 2013; Naseer and Hong, 2013; Naseer et al., 2014; Hong et al., 2015), signal slope (Power et al., 2011, 2012a,b; Hai et al., 2013; Naseer and Hong, 2013; Power and Chau, 2013; Schudlo and Chau, 2014; Hong et al., 2015), signal variance (Tai and Chau, 2009; Holper and Wolf, 2011), amplitude (Naito et al., 2007; Cui et al., 2010b; Bauernfeind et al., 2011; Stangl et al., 2013), skewness (Tai and Chau, 2009; Holper and Wolf, 2011), kurtosis (Tai and Chau, 2009; Holper and Wolf, 2011), and zero crossing (Tai and Chau, 2009).

\section{FILTER COEFFICIENTS}

Some fNIRS-BCI studies have proposed the use of filter coefficients (as classification features) obtained by Kalman filtering
(Abdelnour and Huppert, 2009), recursive least square estimation (Aqil et al., 2012a), and wavelet transform (Khoa and Nakagawa, 2008; Abibullaev et al., 2011; Abibullaev and An, 2012). They assumed that different brain activities will produce different filter coefficients, in which different signals can be classified. This method has been shown to work well, even though no significant classification-accuracy improvement over the heuristic methods has been demonstrated.

\section{GENETIC ALGORITHMS}

Genetic algorithms are an optimization technique that is used to select the most efficient features from a set. Power et al. (2012a) used a genetic algorithm to select features by employing LDA as a fitness function. For more details on genetic algorithms, please see Pernkopf and O'Leary (2001) and Nicolas-Alonso and Gomez-Gil (2012).

Although feature selection is also dependent on individual activities, the mean values and slope values of $\mathrm{HbO}, \mathrm{HbR}$, or $\mathrm{HbT}$ frequently have been used in fNIRS-BCI. Almost half of fNIRSBCI studies have used either the mean value or the slope value of the signal as one of the features for classification. It has been shown that $\mathrm{HbO}$ performs more robustly than $\mathrm{HbR}$ and $\mathrm{HbT}$ for assessing task-related cortical activation (Mihara et al., 2012; Naseer and Hong, 2013; Naseer et al., 2014). Plichta et al. (2006) showed that the retest reliability and stability over time of $\mathrm{HbO}$ signals are higher than those of HbR signals. From the above reasons, feature extraction using $\mathrm{HbO}$ signals is more suitable for classification in ANIRS-BCI.

\section{CLASSIFICATION TECHNIOUES}

Classification techniques are used to identify the different brain signals that are generated by the user. These identified signals are then translated into control commands for application interface purposes. In most existing fNIRS-BCIs, such identification is performed by using classification techniques to discriminate various brain signals based on appropriate features. Classification algorithms, as calibrated by the users through supervised learning during the training phase, are able to detect brain-signal patterns during the testing stage. Some of the commonly used classification methods in ANIRS-BCI are LDA, SVM, HMM, and artificial neural networks (ANN).

\section{LDA}

LDA is the most commonly used classification in ANIRS-BCI studies (see Figure 3). It utilizes discriminant hyperplane(s) to separate data representing two or more classes. Because of its simplicity and low computational requirements, it is highly suitable for online BCI systems. Not surprisingly, it has been used in a number of fNIRS-BCI studies. In LDA, the separating hyperplane is found by seeking such data projection by maximizing the distance between the two classes' means and minimizing the interclass variances. LDA assumes a normal data distribution along with an equal covariance matrix for both classes (Lotte et al., 2007). An LDA algorithm tries to find a vector $v$ in the feature space such that two projected classes 1 and 2 in the $v$ direction can be well separated from each other while maintaining a small variance for each (see Figure 4). This can be accomplished by maximizing the Fisher's criterion given by: 


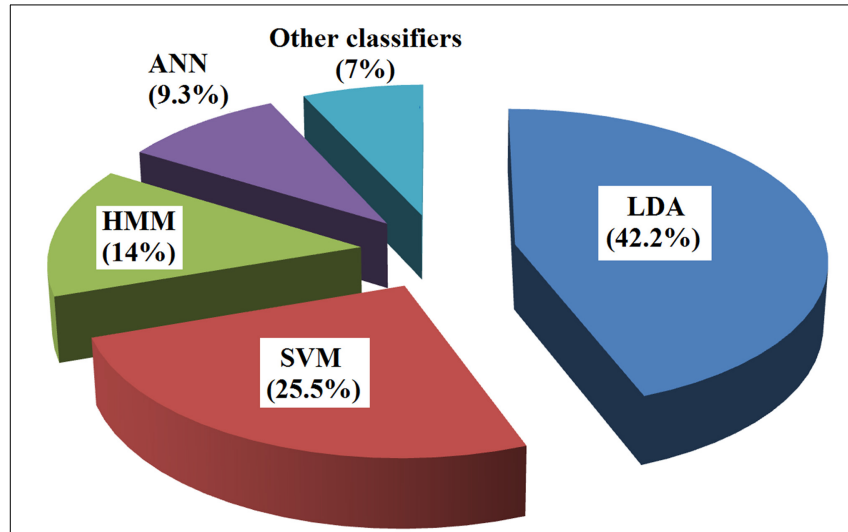

FIGURE 3 | Types of classifiers in fNIRS BCI (from 2004 to 2014).

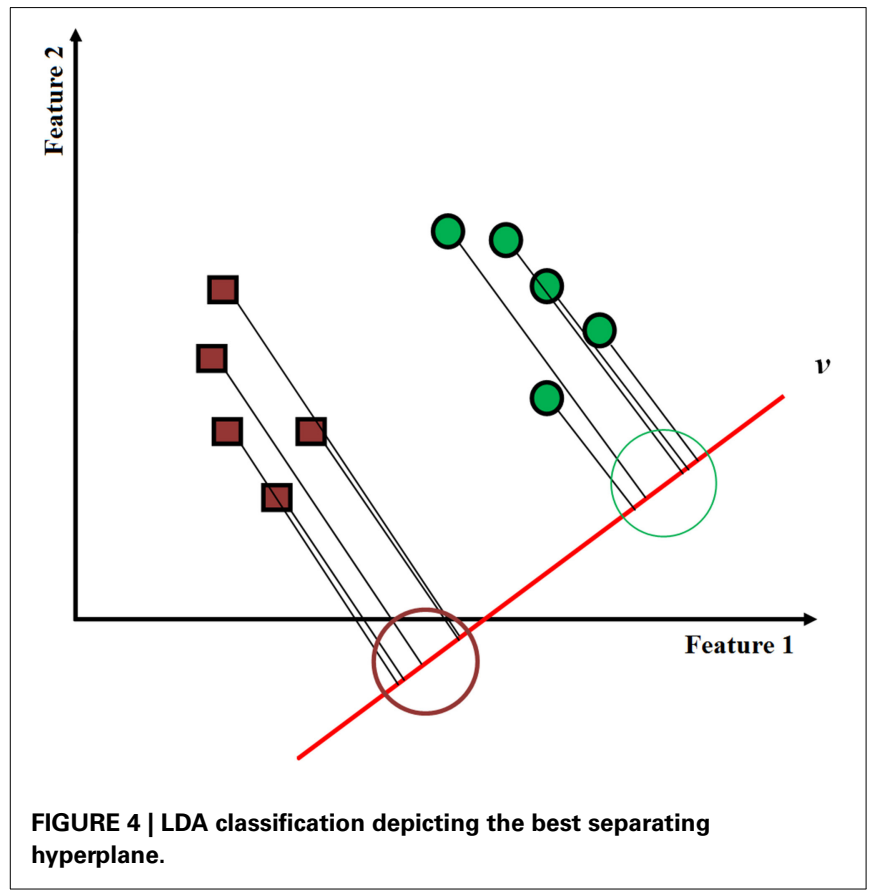

$$
J(v)=\frac{v^{\mathrm{T}} S_{\mathrm{b}} v}{v^{\mathrm{T}} S_{\mathrm{w}} v}
$$

where $S_{b}$ and $S_{w}$ are the between-class and within-class scatter matrices defined as:

$$
\begin{aligned}
& S_{\mathrm{b}}=\left(m_{1}-m_{2}\right)\left(m_{1}-m_{2}\right)^{\mathrm{T}}, \\
& S_{\mathrm{w}}=\sum_{x_{n} \in \mathrm{C} 1}\left(x_{n}-m_{1}\right)\left(x_{n}-m_{2}\right)^{\mathrm{T}}+\sum_{x_{n} \in \mathrm{C} 2}\left(x_{n}-m_{1}\right)\left(x_{n}-m_{2}\right)^{\mathrm{T}}(3)
\end{aligned}
$$

where $m_{1}$ and $m_{2}$ represent the group means of classes $\mathrm{C} 1$ and C2, respectively, and, $x_{n}$ denotes samples. It can be seen that a vector $v$ that satisfies (1) can be reformulated as a generalized eigenvalue problem as:

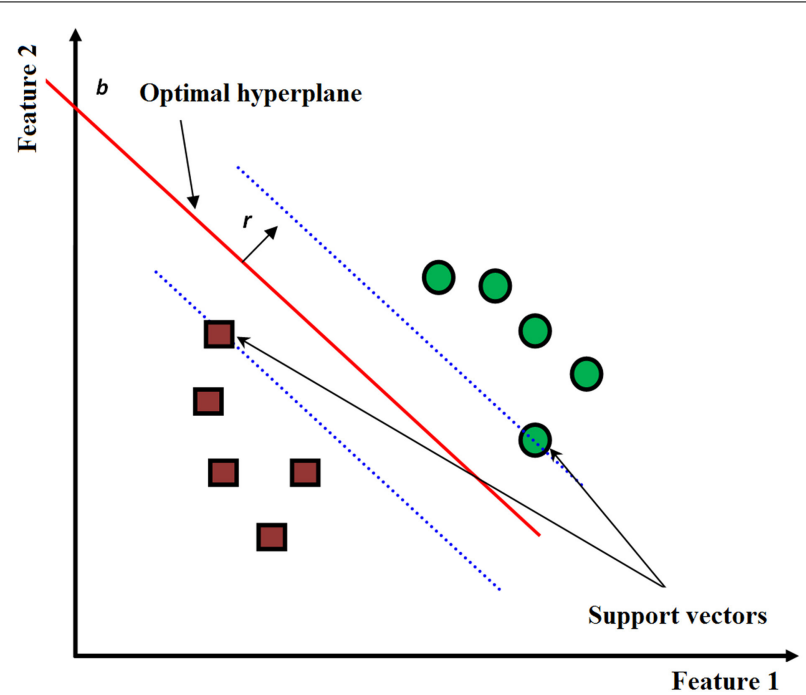

FIGURE 5 | SVM classification illustrating the optimal hyperplane that maximizes the distance from the nearest support vectors.

$$
S_{\mathrm{w}}^{-1} S_{\mathrm{b}} v=\lambda v
$$

The optimal $v$ is then the eigenvector corresponding to the largest eigenvalue of $S_{\mathrm{w}}^{-1} S_{\mathrm{b}}$ or is directly obtained as:

$$
v=S_{\mathrm{w}}^{-1}\left(m_{1}-m_{2}\right)
$$

provided that $S_{\mathrm{w}}$ is non-singular.

Many fNIRS studies have successfully demonstrated the use of LDA for BCI (Luu and Chau, 2009; Bauernfeind et al., 2011; Holper and Wolf, 2011; Power et al., 2011, 2012a,b; Abibullaev and An, 2012; Fazli et al., 2012; Moghimi et al., 2012; Faress and Chau, 2013; Naseer and Hong, 2013; Power and Chau, 2013; Stangl et al., 2013; Kaiser et al., 2014; Naseer et al., 2014; Schudlo and Chau, 2014; Hong et al., 2015).

\section{SVM}

The SVM classifier tries to maximize the distance between the separating hyperplane and the nearest training point(s) (the so-called support vectors) (see Figure 5). The separating hyperplane in the $2 \mathrm{D}$ feature space is given by the equation:

$$
f(x)=r \cdot x+b,
$$

where $r, x \in R^{2}$ and $b \in R^{1}$ (see Figure 5). The optimal solution $r^{*}$ that maximizes the distance between the hyperplane and the nearest training point(s) can be obtained by minimizing the cost function.

$$
J(r, \xi)=\frac{1}{2}\|r\|^{2}+C \cdot \sum_{n=1}^{z} \xi_{n},
$$

while satisfying the constraints: 


$$
\begin{array}{r}
\left(x_{n} \cdot r+b\right) \geq 1-\xi_{n} \text { for } y_{n}=+1, \\
\left(x_{n} \cdot r+b\right) \geq-1+\xi_{n} \text { for } y_{n}=-1, \\
\xi_{n} \geq 0 \forall n,
\end{array}
$$

where $\|r\|^{2}=r^{T} r, C$ is the positive regularization parameter chosen by the user (a large value of $C$ corresponds to a higher penalty for classification errors), $\xi_{\mathrm{n}}$ is the measure of training error, $z$ is the number of misclassified samples, and $y_{n}$ is the class label $(+1$ or -1 in the case of binary classification) for the $n$-th sample.

Since SVM maximizes the distance from the nearest training point(s), it is known to enhance the generalization capabilities. Also, the regularization parameter $C$ allows for accommodating the outliers and therefore reduces errors on the training sets (Burges, 1998). Although SVM is a linear classifier because it uses one or more hyperplanes, it is possible to make SVM with non-linear decision boundaries. This can be done by using kernel functions such as the Gaussian or radial basis functions (known commonly as RBF). Non-linear SVM provides a more flexible decision boundary that can result in an increased classification accuracy. Using the kernel functions might, however, be computationally more demanding.

SVM has been shown to work well in a number of fNIRS-BCI studies (Sitaram et al., 2007; Tai and Chau, 2009; Cui et al., 2010b; Tanaka and Katura, 2011; Abibullaev and An, 2012; Hu et al., 2012; Misawa et al., 2012; Hai et al., 2013; Naseer et al., 2014).

\section{ANN}

ANNs are non-linear classifiers that have been used in a few fNIRS-BCI studies (Abibullaev et al., 2011; Chan et al., 2012; Hai et al., 2013). ANNs were inspired by the fact that the human and animal brains are able to react adaptively to changes in internal and external environments. An appropriate model of the nervous system can produce a similar process in an artificial system. ANNs therefore try to mimic brain activity to solve problems. ANNs are widely used in pattern recognition problems, owing to their post-training capability to recognize sets of training-data-related patterns. ANNs consist of assemblies of several artificial neurons that allow for the drawing of nonlinear decision boundaries. They can be used in several different architectures including multilayer perception, Gaussian classifier, learning vector quantization, RBF neural networks, and others. For more details on these architectures, please see (Anthony and Bartlett, 2009).

\section{HMM}

HMM is a non-linear probabilistic classifier that provides the probability of observing a given set of features that are suitable primarily for classification of time series (Rabiner, 1989). Some fNIRS studies, for example, have successfully demonstrated the feasibility of using HMM for BCI (Sitaram et al., 2007; Power et al., 2010; Falk et al., 2011; Chan et al., 2012; Zimmermann et al., 2013).

Two other classifiers that have been used in fNIRS-BCI are partial least squares discriminant analysis (PLSDA) (Seo et al., 2012) and quadratic discriminant analysis (QDA) (Naito et al., 2007). Although some non-linear classifiers have been shown to increase classification accuracies over those of linear classifiers, the high-speed execution of the linear classifiers has made them the preferred ones for fNIRS-BCI. Almost 45\% of fNIRS-BCI studies have utilized LDA for classification (see Figure 3), due specifically to its fine balance between the classification accuracy and the execution speed.

\section{fNIRS-BCI APPLICATIONS}

In recent years, significant progress has been made in fNIRS-BCI research; however, the applications have been designed mostly for training and demonstration purposes only. fNIRS-BCI has two main drawbacks that have limited its use in real-world applications: a slow information transfer rate, and high error rates. Another problem is the fact that most fNIRS-BCIs are tested in controlled laboratory environments where the user can comfortably concentrate well on mental tasks; whereas in real situations, performance of concentration-dependent mental tasks (e.g., motor imagery, mental arithmetic, etc.) is much more challenging.

\section{NEURO-REHABILITATION}

BCI systems can be used to restore some of the lost motor and/or cognitive functions in individuals with stroke and spinal cord injury. The underlying idea of doing so is the ability of BCI feedback to induce self-regulation of brain activity. EEG, due to its high temporal resolution, has been used in a large number of previous neurofeedback studies (please see Gruzelier, 2013, for a review of EEG-based neurofeedback studies). However, since EEG has the limitations of imprecise localization and inaccessibility of subcortical areas, the hemodynamic activity measured by fMRI has been used in neurofeedback studies to overcome these problems. A comprehensive review of fMRI-based BCI and neurofeedback studies is provided by LaConte (2011) and Weiskopf (2012).

fNIRS is very attractive, in comparison with fMRI, in accessing subcortical brain signals. It is low cost, easy to use, and most of all it is portable. It can be used even in an ambulance. It also has a better temporal resolution than most of fMRI scanners (Huppert et al., 2006). Moreover, fNIRS is less sensitive to motion artifacts because it can be attached (or worn) to the brain or on the body. Given the above points, the potential of use of fNIRS in neurofeedback studies is very high. Mihara et al. (2012) demonstrated the possibility of using fNIRS-based neurofeedback to allow the users to willfully regulate their hemodynamic responses. They also showed that fNIRS-based neurofeedback enhances the hemodynamic correlates corresponding to motor imagery. Further, the same group have also reported similar results for stoke patients (Mihara et al., 2013). Recently, Kober et al. (2014) revealed that fNIRS-based neurofeedback can be used for a long-term training as well, and such repetitive neurofeedback can induce specific and focused brain activation: In contrast, sham feedback has led to diffuse brain activation patterns over broader brain areas. One important disadvantage of using hemodynamics (either fMRI or fNIRS) for neurofeedback is the inherent delay in its response, which makes the generation of commands slow compared to EEG. However, in the case of fNIRS, this kind of disadvantage can be solved if the initial dip (i.e., the phenomenon that $\mathrm{HbO}$ decreases 
and $\mathrm{HbR}$ increases with neural firing) can be measured (instead of hemodynamics).

\section{COMMUNICATION}

The primary application of $\mathrm{BCI}$ is to serve as a means of communication for people with motor disorders such as ALS, spinal cord injury and/or who are suffering from a persistent LIS. Naito et al. (2007) and Naseer et al. (2014) developed an fNIRS-BCI system for binary communication based on activations from the prefrontal area. The subjects were required to perform a specific task such as mental arithmetic or music imagery to increase the cognitive load and, thereby, respond "yes" or to remain relax and, thus, respond "no" to the given question. The average accuracies obtained by Naseer et al. (2014) with online classification were approximately $82 \%$. Sitaram et al. (2007) proposed an ANIRS-BCI-based online word speller. Their system involves using right-hand and left-hand motor imagery to move a cursor on a two-dimensional to select letters.

\section{MOTOR RESTORATION/REHABILITATION}

Another important application of fNIRS-BCI is the restoration of movement capability for people with motor disabilities. The control commands generated by a BCI system can be used to control a prosthetic limb or a wheelchair. It is desirable to have a portable system for these applications so that the user can move freely. Also these applications, for safety purposes, cannot afford high error rates, and must be fast enough to provide real-time control. Several fNIRS-BCI studies have tried to improve classification accuracies and information transfer rates (Shin and Jeong, 2014). Using neurofeedback, induction of neuroplasticity of selected brain areas which has the potential to improve cognitive performance, also can be accomplished.

\section{OTHER APPLICATIONS}

Other applications of fNIRS-BCI include environment control and entertainment. Environment control applications (for instance, remote control, lights and temperature control) are very useful for motor-disabled people. Recently, BCI has also been used for healthy individuals' entertainment purposes, although this is not a main priority of $\mathrm{BCI}$ research. The feasibility of braincontrolled video games has been demonstrated using EEG-BCI; however, no such fNIRS-based application has been introduced to date. For training purposes though, such games might be useful.

Table 1 provides a summary of most studies published from 2004 to 2014 that demonstrated important roles in brain-signalacquisition, signal pre-processing, feature-selection, and classification stages for fNIRS-BCI.

\section{FUTURE PROSPECTS OF FNIRS-BCI}

Given the advantages (non-invasive, cheap, portable, and silent), the use of ANIRS for BCI purposes is more suitable than fMRI. Furthermore, its use is easier than EEG that uses wet electrodes. A limitation of using fNIRS for BCI is that the information transfer rate is limited by the inherent delay in the hemodynamic response. However, the detections of the fast optical response (Gratton et al., 2006; Hu et al., 2011) and the initial dip (Akin et al., 2006; Yoshino and Kato, 2012) have been demonstrated, which can offer faster information transfer rate and better control. Since the speed of EEG can be utilized, the authors believe that the future of non-invasive, portable and wearable BCIs lies in the use of hybrid EEG-fNIRS systems, as it has shown to work superior to EEG-BCIs and fNIRS-BCIs alone (Fazli et al., 2012; Kaiser et al., 2014; Khan et al., 2014; Koo et al., 2014). The reason for using a hybrid or combined fNIRS-EEG system is that it either improves the classification accuracy or increases the number of control commands for BCI. This can be done by extracting some relevant features from fNIRS and combining them with EEG system. Fazli et al. (2012) demonstrated significantly enhanced performance, in terms of classification accuracy, by combined feature sets from both fNIRS and EEG. Tomita et al. (2014) showed that an optimal time slot for command generation can be estimated using indications from fNIRS signals in hybrid fNIRS-EEG. Khan et al. (2014) demonstrated an efficient control strategy for active BCI by placing fNIRS and EEG at different brain locations. Koo et al. (2014) have also shown that the self-paced motor imagery can be detected more efficiently using a hybrid fNIRS-EEG system. Since the information contents of EEG and fNIRS are very distinctive, the hybrid fNIRS-EEG system has a strong potential for future neurorehabilitation and neurofeedback applications.

\section{CONCLUSIONS}

In this paper, we have reviewed the state-of-the-art of fNIRSbased BCI systems, discussing all the procedures appearing in the standard BCI. Several different brain activities have been used for fNIRS-BCI, including, most commonly, those from the motor and prefrontal cortices. Motor cortex activities such as motor execution and motor imagery have been shown to work well and, indeed, are useful from the neurorehabilitation perspective. Prefrontal activities, on the other hand, offer the advantages of being free from artifacts due to hair. Both, despite of their drawbacks, have been shown to work well for fNIRS-BCI purposes. Use of other brain-imaging modalities, such as EEG in combination with fNIRS in a hybrid fashion, has been shown to effectively improve BCI performance. Such hybrid systems can acquire brain signals from the same as well as different brain areas, thereby increasing the number of control commands.

Different signal-processing and noise-removal methods including band-pass filtering, ICA, principle component analysis, wavelet transform and adaptive-filtering-based methods have been discussed. Because band-pass filters are simple and incur only low computational costs, they are still mostly used in fNIRS BCI.

BCI-applied classification algorithms must be both accurate and fast. Although SVM, hidden Markov models, and artificial neural networks provide good classification accuracies, the linear discriminant analysis (in its simple structure) has a low computational cost and also provides a good performance in classification accuracy.

Considering all these points, it is concluded that there is much room for future fNIRS-BCI research, particularly in its applications. Although fNIRS-BCI applications for communication and control have been demonstrated in a number of studies, no commercial fNIRS-BCI application currently is available. All of the relevant research trends predict that interest in ANIRS-BCI will 


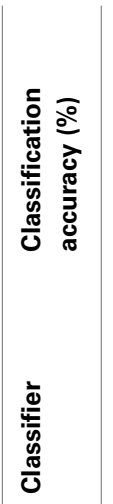

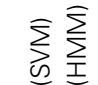

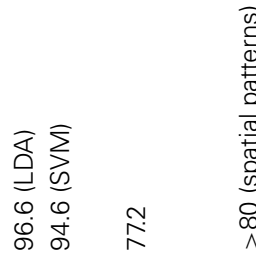

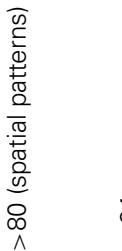

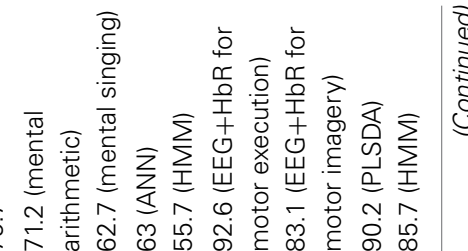

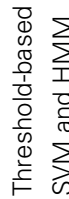

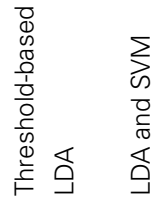

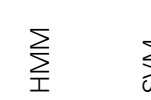

$\sum_{\infty}$

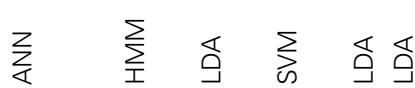

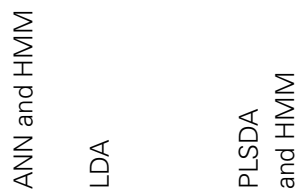

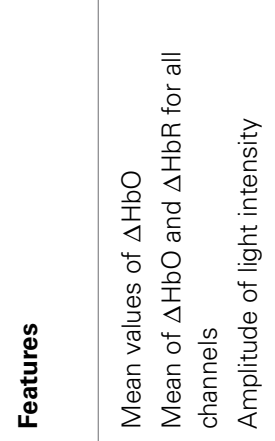

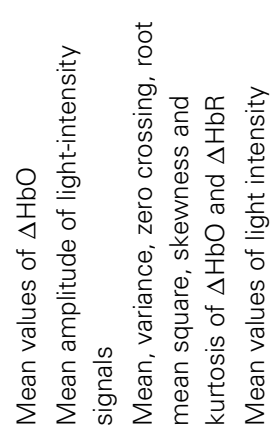

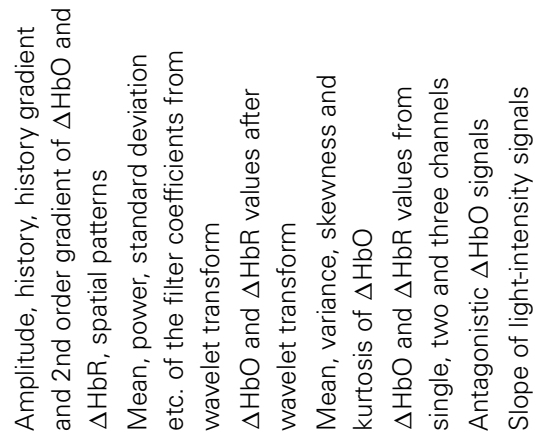

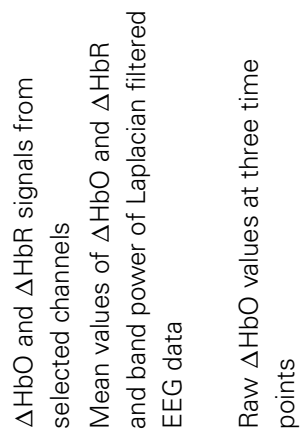

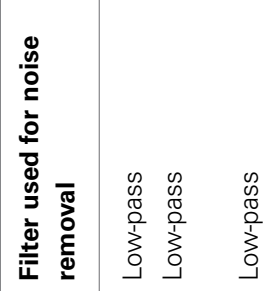

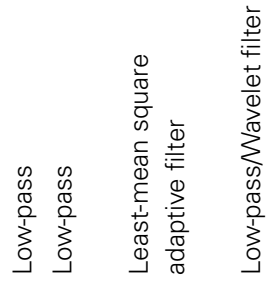

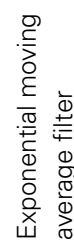

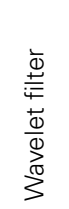

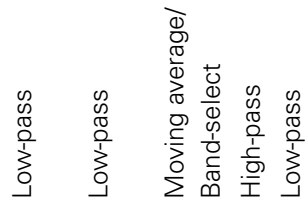

$\begin{array}{ll}0 & 0 \\ 0 & 0 \\ 0 & 0 \\ 0 & 0 \\ 0 & 1 \\ 3 & 3 \\ 3 & 0\end{array}$

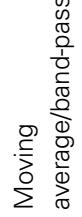

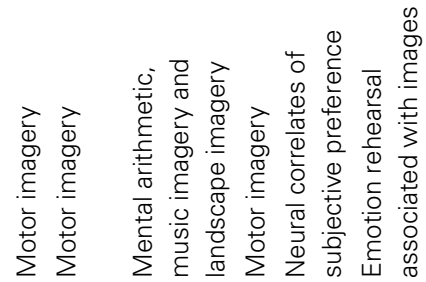

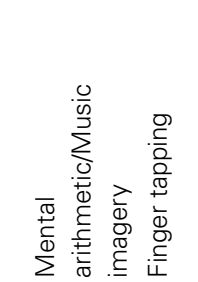

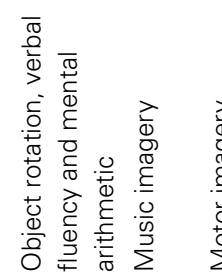

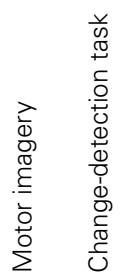

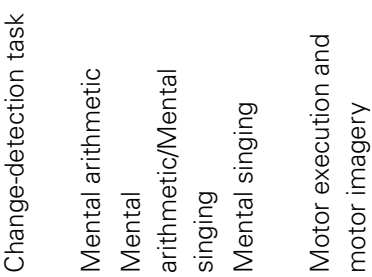

ডั

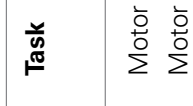
(1)

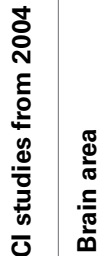

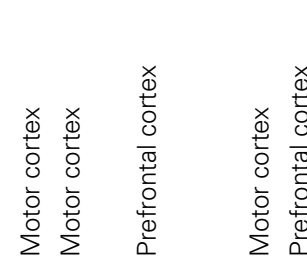

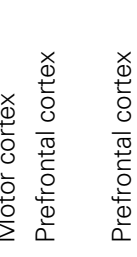

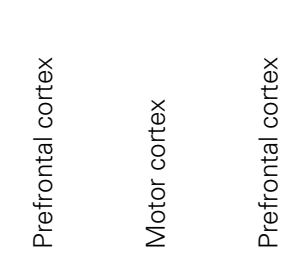
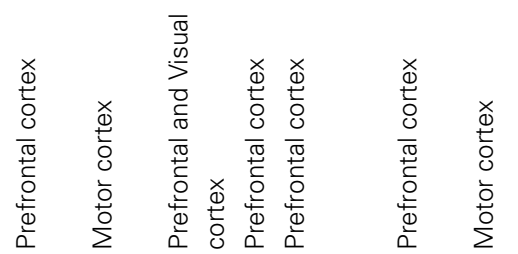

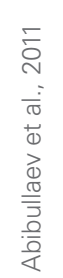

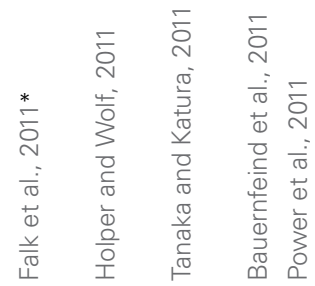

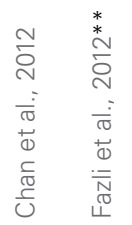

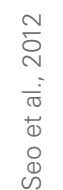




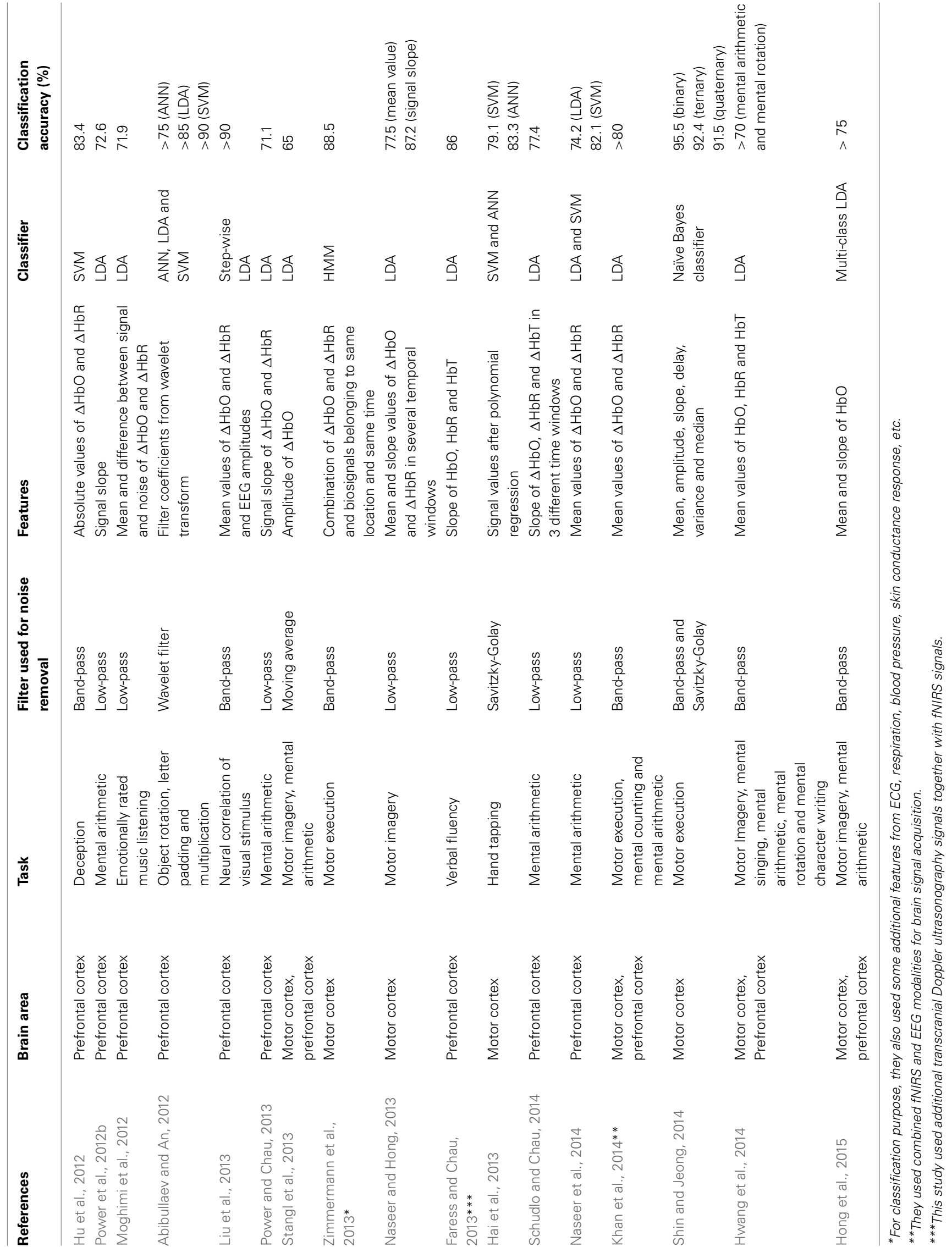


continue to grow. In the near future, several breakthroughs via bundled-type fNIRS probes, hybrid EEG-fNIRS, and detection of the initial dip are expected.

\section{ACKNOWLEDGMENTS}

This work was supported by the National Research Foundation of Korea under the Ministry of Science, ICT and Future Planning, Korea (grant no. NRF-2014R1A2A1A10049727).

\section{REFERENCES}

Abdelnour, A. F., and Huppert, T. (2009). Real-time imaging of human brain function by near-infrared spectroscopy using an adaptive general linear model. Neuroimage 46, 133-143. doi: 10.1016/j.neuroimage.2009. 01.033

Abibullaev, B., and An, J. (2012). Classification of frontal cortex hemodynamic responses during cognitive tasks using wavelet transforms and machine learning algorithms. Med. Eng. Phys. 34, 1394-1410. doi: 10.1016/j.medengphy.2012.01.002

Abibullaev, B., An, J., and Moon, J. I. (2011). Neural network classification of brain hemodynamic responses from four mental tasks. Int. J. Optomechatronics 5 , 340-359. doi: 10.1080/15599612.2011.633209

Adhika, D. R., Hazrati, M. K., and Hofmann, U. G. (2012). An experimental setup for brain activity measurement based on near infrared spectroscopy. Biomed. Tech. 57, 609-612. doi: 10.1515/bmt-2012-4487

Akin, A., Bilensoy, D., Emir, U. E., Gulsoy, M., Candansayar, S., and Bolay, H. (2006). Cerebrovascular dynamics in patients with migraine: near-infrared spectroscopy study. Neurosci. Lett. 400, 86-91. doi: 10.1016/j.neulet.2006.02.016

Anthony, M., and Bartlett, P. L. (2009). Neural Network Learning: Theoretical Foundations. New York, NY: Cambridge University Press.

Aqil, M., Hong, K.-S., Jeong, M.-Y., and Ge, S. S. (2012a). Cortical brain imaging by adaptive filtering of NIRS signals. Neurosci. Lett. 514, 35-41. doi 10.1016/j.neulet.2012.02.048

Aqil, M., Hong, K.-S., Jeong, M.-Y., and Ge, S. S. (2012b). Detection of eventrelated hemodynamic response to neuroactivation by dynamic modeling of brain activity. Neuroimage 63, 553-568. doi: 10.1016/j.neuroimage.2012.07.006

Ayaz, H., Shewokis, P. A., Bunce, S., Izzetoglu, K., Willems, B., and Onaral, B. (2012). Optical brain monitoring for operator training and mental workload assessment. Neuroimage 59, 36-47. doi: 10.1016/j.neuroimage.2011.06.023

Ayaz, H., Shewokis, P., Bunce, S., Schultheis, M., and Onaral, B. (2009). "Assessment of cognitive neural correlates for a functional near infrared-based brain computer interface system," in Foundations of Augmented Cognition. Neuroergonomics and Operational Neuroscience, eds D. D. Schmorrow, I. V. Estabrooke, and M. Grootjen (Heidelberg: Springer-Verlag), 699-708.

Bauernfeind, G., Leeb, R., Wriessnegger, S. C., and Pfurtscheller, G. (2008) Development, set-up and first results for a one-channel near-infrared spectroscopy system. Biomed. Tech. 53, 36-43. doi: 10.1515/BMT.2008.005

Bauernfeind, G., Scherer, R., Pfurtscheller, G., and Neuper, C. (2011). Single-trial classification of antagonistic oxyhemoglobin responses during mental arithmetic. Med. Biol. Eng. Comput. 49, 979-984. doi: 10.1007/s11517-011-0792-5

Bhutta, M. R., Hong, K.-S., Kim, B.-M., Hong, M. J., Kim, Y.-H., and Lee, S.-H (2014). Note: three wavelengths near-infrared spectroscopy system for compensating the light absorbance by water. Rev. Sci. Intrum. 85:026111. doi: $10.1063 / 1.4865124$

Boas, D. A., Chen, K., Grebert, D., and Franceschini, M. A. (2004b). Improving the diffuse optical imaging spatial resolution of the cerebral hemodynamic response to brain activation in humans. Opt. Lett. 29, 1506-1508. doi: 10.1364/OL.29.001506

Boas, D. A., Dale, A. M., and Franceschini, M. A. (2004a). Diffuse optical imaging of brain activation: approaches to optimizing image sensitivity, resolution, and accuracy. Neuroimage 23, S275-S288. doi: 10.1016/j.neuroimage.2004.07.011

Buch, E., Weber, C., Cohen, L. G., Braun, C., Dimyan, M. A., Ard, T., et al. (2008). Think to move: a neuromagnetic brain-computer interface (BCI) system for chronic stroke. Stroke 39, 910-917. doi: 10.1161/STROKEAHA.107.505313

Burges, C. J. C. (1998). A tutorial on support vector machines for pattern recognition. Knowl. Discov. Data Min. 2, 121-167. doi: 10.1023/A:1009715923555

Chan, J., Power, S., and Chau, T. (2012). Investigating the need for modeling temporal dependencies in a brain-computer interface with real-time feedback based on near infrared spectra. J. Near Infrared Spectrosc. 20, 107-116. doi: $10.1255 /$ inirs. 971

Cooper, R. J., Selb, J., Gagnon, L., Phillip, D., Schytz, H. W., Iversen, H. K., et al. (2012). A systematic comparison of motion artifact correction techniques for functional near-infrared spectroscopy. Front. Neurosci. 6:147. doi: $10.3389 /$ fnins.2012.00147

Coyle, S. M., Ward, T. E., and Markham, C. M. (2007). Brain-computer interface using a simplified functional near-infrared spectroscopy system. J. Neural Eng. 4, 219-226. doi: 10.1088/1741-2560/4/3/007

Coyle, S., Ward, T., Markham, C., and McDarby, G. (2004). On the suitability of near-infrared (NIR) systems for next-generation brain-computer interfaces. Physiol. Meas. 25, 815-822. doi: 10.1088/0967-3334/25/4/003

Cui, X., Bray, S., and Reiss, A. L. (2010a). Functional near infrared spectroscopy (NIRS) signal improvement based on negative correlation between oxygenated and deoxygenated hemoglobin dynamics. Neuroimage 49, 3039-3046. doi: 10.1016/j.neuroimage.2009.11.050

Cui, X., Bray, S., and Reiss, A. L. (2010b). Speeded near-infrared spectroscopy (NIRS) response detection. PLoS ONE 5:e15474. doi: 10.1371/journal.pone.0015474

Delpy, D. T., Cope, M., van der Zee, P., Arridge, S., Wray, S., and Wyatt, J. (1988). Estimation of optical pathlength through tissue from direct time of flight measurement. Phys. Med. Biol. 33, 1433-1442. doi: 10.1088/0031-9155/33/12/008

Falk, T. H., Guirgis, M., Power, S., and Chau, T. (2011). Taking NIRS-BCIs outside the lab: towards achieving robustness against environment noise. IEEE Trans. Neural Syst. Rehabil. Eng. 19, 136-146. doi: 10.1109/TNSRE.2010.2078516

Faress, A., and Chau, T. (2013). Towards a multimodal brain-computer interface: combining fNIRS and fTCD measurements to enable higher classification accuracy. Neuroimage 77, 186-194. doi: 10.1016/j.neuroimage.2013.03.028

Fazli, S., Mehnert, J., Steinbrink, J., Curio, G., Villringer, A., Muller, K. R., et al. (2012). Enhanced performance by a hybrid NIRS-EEG brain-computer interface. Neuroimage 59, 519-529. doi: 10.1016/j.neuroimage.2011.07.084

Fekete, T., Rubin, D., Carlson, J. M., and Mujica-Parodi, L. R. (2011). The NIRS analysis package: noise reduction and statistical inference. PLoS ONE 6:e24322. doi: 10.1371/journal.pone.0024322

Ferrari, M., Giannini, I., Sideri, G., and Zanette, E. (1985). Continuous non invasive monitoring of human brain by near infrared spectroscopy. Adv. Exp. Med. Biol. 191, 873-882. doi: 10.1007/978-1-4684-3291-6_88

Ferrari, M., Mottola, L., and Quaresima, V. (2004). Principles, techniques, and limitations of near infrared spectroscopy. Can. J. Appl. Physiol. 29, 463-487. doi: 10.1139/h04-031

Franceschini, M. A., Joseph, D. K., Huppert, T. J., Diamond, S. G., and Boas, D. A. (2006). Diffuse optical imaging of the whole head. J. Biomed. Opt. 11:054007. doi: $10.1117 / 1.2363365$

Funane, T., Atsumori, H., Katura, T., Obata, A. N., Sato, H., Tanikawa, Y., et al. (2014). Quantitative evaluation of deep and shallow tissue layers' contribution to fNIRS signal using multi-distance optodes and independent component analysis. Neuroimage 85, 150-165. doi: 10.1016/j.neuroimage.2013.02.026

Gagnon, L., Yucel, M. A., Dehaes, M., Cooper, R. J., Perdue, K. L., Selb, J., et al. (2012). Quantification of the cortical contribution to the NIRS signal over the motor cortex using concurrent NIRS-fMRI measurements. Neuroimage 59, 3933-3940. doi: 10.1016/j.neuroimage.2011.10.054

Gratton, G., Brumback, C. R., Gordon, B. A., Pearson, M. A., Low, K. A., and Fabiani, M. (2006). Effects of measurement method, wavelength, and sourcedetector distance on the fast optical signal. Neuroimage 32, 1576-1590. doi: 10.1016/j.neuroimage.2006.05.030

Gruzelier, J. H. (2013). EEG-neurofeedback for optimising performance. I: a review of cognitive and affective outcome in healthy participants. Neurosci. Biobehav. Rev. 44, 124-141. doi: 10.1016/j.neubiorev.2013.09.015

Hai, N. T., Cuong, N. Q., Khoa, T. Q. D., and Toi, V. V. (2013). Temporal hemodynamic classification of two hands tapping using functional near-infrared spectroscopy. Front. Hum. Neurosci. 7:516. doi: 10.3389/fnhum.2013.00516

Hammer, E. M., Kaufmann, T., Kleih, S. C., Blankertz, B., and Kubler, A. (2014). Visuo-motor coordination ability predicts performance with brain-computer interfaces controlled by modulation of sensorimotor rhythms (SMR). Front. Hum. Neurosci. 8:574. doi: 10.3389/fnhum.2014. 00574

Holper, L., and Wolf, M. (2011). Single-trial classification of motor imagery differing in task complexity: a functional near-infrared spectroscopy study. J. Neuroeng. Rehabil. 8:34. doi: 10.1186/1743-0003-8-34 
Hong, K.-S., Naseer, N., and Kim, Y.-H. (2015). Classification of prefrontal and motor cortex signals for three-class fNIRS-BCI. Neurosci. Lett. 587, 87-92. doi: 10.1016/j.neulet.2014.12.029

Hong, K.-S., and Nguyen, H.-D. (2014). State-space models of impulse hemodynamic responses over motor, somatosensory, and visual cortices. Biomed. Opt. Express 5, 1778-1798. doi: 10.1364/BOE.5.001778

Hoshi, Y., Onoe, H., Watanabe, Y., Andersson, J., Bergstrom, M., Lilja, A., et al. (1994). Non-synchronous behavior of neuronal-activity, oxidative-metabolism and blood-supply during mental tasks in man. Neurosci. Lett. 172, 129-133. doi: 10.1016/0304-3940(94)90679-3

Hoshi, Y., and Tamura, M. (1997). Near-infrared optical detection of sequential brain activation in the prefrontal cortex during mental tasks. Neuroimage 5, 292-297. doi: 10.1006/nimg.1997.0270

Hu, X.-S., Hong, K.-S., and Ge, S. S. (2011). Recognition of stimulus-evoked neuronal optical response by identifying chaos levels of near-infrared spectroscopy time series. Neurosci. Lett. 504, 115-120. doi: 10.1016/j.neulet.2011.09.011

$\mathrm{Hu}, \mathrm{X} .-\mathrm{S}$. , Hong, K.-S., and Ge, S. S. (2012). fNIRS-based online deception decoding. J Neural Eng. 9:026012. doi: 10.1088/1741-2560/9/2/026012

$\mathrm{Hu}$, X.-S., Hong, K.-S., and Ge, S. S. (2013). Reduction of trial-to-trial variations in functional near-infrared spectroscopy signals by accounting for resting-state functional connectivity. J. Biomed. Opt. 18:017003. doi: 10.1117/1.JBO.18.1.017003

Hu, X.-S., Hong, K.-S., Ge, S. S., and Jeong, M.-Y. (2010). Kalman estimator- and general linear model-based on-line brain activation mapping by near-infrared spectroscopy. Biomed. Eng. Online 9:82. doi: 10.1186/1475-925X-9-82

Huppert, T. J., Diamond, S. G., Fransceshini, M. A., and Boas, D. A. (2009). HomER: a review of time-series analysis methods for near-infrared spectroscopy of the brain. Appl. Opt. 48, D280-D298. doi: 10.1364/AO.48.00D280

Huppert, T. J., Hoge, R. D., Diamond, S. G., Franceschini, M. A., and Boas, D. A. (2006). A temporal comparison of BOLD, ASL, and NIRS hemodynamic responses to motor stimuli in adult humans. Neuroimage 29, 368-382. doi: 10.1016/j.neuroimage.2005.08.065

Hwang, H.-J., Kim, S., Choi, S., and Im, C.-H. (2013). EEG-based brain-computer interfaces: a thorough literature survey. Int. J. Hum. Comp. Int. 29, 814-826. doi: 10.1080/10447318.2013.780869

Hwang, H.-J., Lim, J.-H., Kim, D.-W., and Im, C.-H. (2014). Evaluation of various mental task combinations for near-infrared spectroscopy-based braincomputer interfaces. J. Biomed. Opt. 19:077005. doi: 10.1117/1.JBO.19.7.077005

Izzetoglu, M., Devaraj, A., Bunce, S., and Onaral, B. (2005). Motion artifact cancellation in NIR spectroscopy using Wiener filtering. IEEE Trans. Biomed. Eng. 52, 934-938. doi: 10.1109/TBME.2005.845243

Jobsis, F. F. (1977). Noninvasive, infrared monitoring of cerebral and myocardial oxygen sufficiency and circulatory parameters. Science 198, 1264-1267. doi: 10.1126/science.929199

Kaiser, V., Gauernfeind, G., Kreilinger, A., Kaufmann, T., Kubler, A., Neuper, C., et al. (2014). Cortical effects of user training in a motor imagery based braincomputer interface measured by fNIRS and EEG. Neuroimage 85, 432-444. doi: 10.1016/j.neuroimage.2013.04.097

Kamran, M. A., and Hong, K.-S. (2013). Linear parameter-varying model and adaptive filtering technique for detecting neuronal activities: an fNIRS study. J. Neural Eng. 10:056002. doi: 10.1088/1741-2560/10/5/056002

Kamran, M. A., and Hong, K.-S. (2014). Reduction of physiological effects in fNIRS waveforms for efficient brain-state decoding. Neurosci. Lett. 580, 130-136. doi: 10.1016/j.neulet.2014.07.058

Kato, T., Kamei, A., Takashima, S., and Ozaki, T. (1993). Human visual cortical function during photic stimulation monitoring by means of near-infrared spectroscopy. J. Cereb. Blood Flow Metab. 13, 516-520. doi: 10.1038/jcbfm. 1993.66

Khan, M. J., Hong, M. J., and Hong, K.-S. (2014). Decoding of four movement directions using hybrid NIRS-EEG brain-computer interface. Front. Hum. Neurosci. 8:244. doi: 10.3389/fnhum.2014.00244

Khoa, T. Q. D., and Nakagawa, M. (2008). Functional near-infrared spectroscope for cognition brain tasks by wavelets analysis and neural networks. Int. J. Biol. Life Sci. 4, 28-33. doi: 10.1186/1753-4631-2-3

Kim, J.-Y., Kang, H.-C., Cho, J.-H., Lee, J.-H., Kim, H.-D., and Im, C.-H. (2014). Combined use of multiple computational intracranial EEG analysis techniques for the localization of epileptogenic zones in Lennox-Gastaut syndrome. Clin. EEG Neurosci. 45, 169-178. doi: 10.1177/1550059413495393

Kirilina, E., Jelzow, A., Heine, A., Niessing, M., Wabnitz, H., Brühl, R., et al. (2012). The physiological origin of task-evoked systemic artefacts in functional near infrared spectroscopy. Neuroimage 61, 70-81. doi: 10.1016/j.neuroimage.2012.02.074

Kirilina, E., Yu, N., Jelzow, A., Wabnitz, H., Jacobs, A. M., and Tachtsidis, I. (2013). Identifying and quantifying main components of physiological noise in functional near infrared spectroscopy on the prefrontal cortex. Front. Hum. Neurosci. 7:864. doi: 10.3389/fnhum.2013.00864

Kleih, S. C., and Kubler, A. (2013). Empathy, motivation, and P300-BCI performance. Front. Hum. Neurosci. 7:642. doi: 10.3389/fnhum.2013.00642

Ko, K. E., and Sim, K. B. (2013). Harmony search-based hidden Markov model optimization for online classification of single trial EEGs during motor imagery tasks. Int. J. Control. Autom. 11, 608-613. doi: 10.1007/s12555-012-0035-z

Kober, S. E., Wood, G., Kurzmann, J., Friedrich, E. V., Stangl, M., Wippel, T., et al. (2014). Near-infrared spectroscopy based neurofeedback training increases specific motor imagery related cortical activation compared to sham feedback. Biol. Psychol. 95, 21-30. doi: 10.1016/j.biopsycho.2013.05.005

Kohno, S., Miyai, I., Seiyama, A., Oda, I., Ishikawa, A., Tsuneishi, S., et al. (2007) Removal of the skin blood flow artifact in functional near-infrared spectroscopic imaging data through independent component analysis. J. Biomed. Opt. 12:062111. doi: 10.1117/1.2814249

Koo, B., Lee, H.-G., Nam, Y., Kang, H., Koh, C.-S., Shin, H.-C., et al. (2014). A hybrid NIRS-EEG system for self-paced brain computer interface with online motor imagery. J. Neurosci. Methods. doi: 10.1016/j.jneumeth.2014.04.016. [Epub ahead of print].

LaConte, S. M. (2011). Decoding fMRI brain states in real-time. Neuroimage 56, 440-454. doi: 10.1016/j.neuroimage.2010.06.052

Liu, Y., Ayaz, H., Curtin, A., and Onarall, B. (2013). "Towards a hybrid P300based BCI using simultaneous ANIR and EEG," in Foundations of Augmented Cognition, eds D. Schmorrow and C. Fidopiastis (Heidelberg: Springer-Verlag), 335-344.

Lotte, F., Congedo, M., Lecuyer, A., Lamarche, F., and Arnaldi, B. (2007). A review of classification algorithms for EEG-based brain-computer interfaces. J. Neural Eng. 4:R1. doi: 10.1088/1741-2560/4/2/R01

Luu, S., and Chau, T. (2009). Decoding subjective preferences from single-trial near-infrared spectroscopy signals. J. Neural Eng. 6:016003. doi: 10.1088/1741$2560 / 6 / 1 / 016003$

McCormick, P. W., Stewart, M., Lewis, G., Dujovny, M., and Ausman, J. I. (1992). Intracerebral penetration of infrared light: technical note. J. Neurosurg. 76, 315-318. doi: 10.3171/jns.1992.76.2.0315

Mellinger, J., Schalk, G., Braun, C., Preissl, H., Rosenstiel, W., Birbaumer, N., et al. (2007). An MEG-based brain-computer interface (BCI). Neuroimage 36, 581-593. doi: 10.1016/j.neuroimage.2007.03.019

Mihara, M., Hattori, N., Hatakenaka, M., Yagura, H., and Kawano, T. (2013). Nearinfrared spectroscopy-mediated neurofeedback enhances efficacy of motor imagery-based training in poststroke victims a pilot study. Stroke 44, 1091-1098. doi: 10.1161/STROKEAHA.111.674507

Mihara, M., Miyai, I., Hattori, N., Hatakenaka, M., Yagura, H., Kawano, T., et al. (2012). Neurofeedback using real-time near-infrared spectroscopy enhances motor imagery related cortical activation. PLOS ONE 7:e32234. doi: 10.1371/journal.pone.0032234

Misawa, T., Takano, S., Shimokawa, T., and Hirobayashi, S. (2012). A braincomputer interface for motor assist by the prefrontal cortex. Electron. Comm. Jpn. 95, 1-8. doi: 10.1002/ecj.11426

Moghimi, S., Kushki, A., Power, S., Guerguerian, A. M., and Chau, T. (2012). Automatic detection of a prefrontal cortical response to emotionally rated music using multi-channel near-infrared spectroscopy. J. Neural Eng. 9:026022. doi: $10.1088 / 1741-2560 / 9 / 2 / 026022$

Nagaoka, T., Sakatani, K., Awano, T., Yokose, N., Hoshino, T., Murata, Y., et al. (2010). "Development of a new rehabilitation system based on a braincomputer interface using near-infrared spectroscopy," in Experimental Medicine and Biology, Oxygen Transport in Tissue XXXI, eds E. Takashi and D. F. Bruley (New York, NY: Springer), 497-503.

Naito, M., Michioka, Y., Ozawa, K., Ito, Y., Kiguchi, M., and Kanazawa, T. (2007). A communication means for totally locked-in ALS patients based on changes in cerebral blood volume measured with near-infrared light. IEICE T. Inf. Syst. E90D, 1028-1037. doi: 10.1093/ietisy/e90-d.7.1028

Naseer, N., and Hong, K.-S. (2013). Classification of functional near-infrared spectroscopy signals corresponding to the right- and left-wrist motor imagery for development of a brain-computer interface. Neurosci. Lett. 553, 84-49. doi: 10.1016/j.neulet.2013.08.021 
Naseer, N., Hong, M. J., and Hong, K.-S. (2014). Online binary decision decoding using functional near-infrared spectroscopy for the development of braincomputer interface. Exp. Brain Res. 232, 555-564. doi: 10.1007/s00221-0133764-1

Nicolas-Alonso, L. F., and Gomez-Gil, J. (2012). Brain computer interfaces, a review. Sensors 2, 1211-1279. doi: 10.3390/s120201211

Pernkopf, F., and O'Leary, P. (2001). "Feature selection for classification using genetic algorithms with a novel encoding," in Computer Analysis of Images and Patterns, ed W. Skarbek (Heidelberg: Springer-Verlag), 161-168.

Plichta, M., Herrmann, M., Baehne, C., Ehlis, A.-C., Richter, M., Pauli, P., et al. (2006). Event-related functional near-infrared spectroscopy (fNIRS): are the measurements reliable? Neuroimage 31, 116-124. doi: 10.1016/j.neuroimage.2005.12.008

Power, S., and Chau, T. (2013). Automatic single-trial classification of prefrontal hemodynamic activity in an individual with Duchenne muscular dystrophy. Dev. Neurorehabil. 16, 67-72. doi: 10.3109/17518423.2012.718293

Power, S. D., Falk, T. H., and Chau, T. (2010). Classification of prefrontal activity due to mental arithmetic and music imagery using hidden Markov models and frequency domain near-infrared spectroscopy. J. Neural Eng. 7:026002. doi: 10.1088/1741-2560/7/2/026002

Power, S. D., Khushki, A., and Chau, T. (2012a). Automatic single trial discrimination of mental arithmetic, mental singing and the no-control state from the prefrontal activity: towards a three-state NIRS-BCI. BMC Res. Notes 5:141. doi: 10.1186/1756-0500-5-141

Power, S. D., Kushki, A., and Chau, T. (2011). Towards a system-paced near-infrared spectroscopy brain-computer interface: differentiating prefrontal activity due to mental arithmetic and mental singing from the no-control state. J. Neural Eng. 8:066004. doi: 10.1088/1741-2560/8/6/066004

Power, S. D., Kushki, A., and Chau, T. (2012b). Intersession consistency of singletrial classification of the prefrontal response to mental arithmetic and the nocontrol state by NIRS. PLoS ONE 7:e37791. doi: 10.1371/journal.pone.0037791

Rabiner, L. R. (1989). A tutorial on hidden Markov models and selected applications in speech recognition. Proc. IEEE 77, 257-286. doi: 10.1109/5. 18626

Rea, M., Rana, M., Lugato, N., Terekhin, P., Gizzi, L., Brotz, D., et al. (2014). Lower limb movement preparation in chronic stroke: a pilot study toward an fNIRS-BCI for gait rehabilitation. Neurorehabil. Neural Repair 28, 564-575. doi: $10.1177 / 1545968313520410$

Saager, R. B., and Berger, A. J. (2005). Direct characterization and removal of interfering absorption trends in two-layer turbid media. J. Opt. Soc. Am. A Opt. Image Sci. Vis. 22, 1874-1882. doi: 10.1364/JOSAA.22.001874

Saager, R. B., Telleri, N. L., and Berger, A. J. (2011). Two-detector corrected near infrared spectroscopy (C-NIRS) detects hemodynamic activation responses more robustly than single-detector NIRS. Neuroimage 55, 1679-1685. doi: 10.1016/j.neuroimage.2011.01.043

Sagara, K., and Kido, K. (2012). Evaluation of a 2-channel NIRS-based optical brain switch for motor disabilities' communication tools. IEICE T. Inf. Syst. E95D, 829-834. doi: 10.1587/transinf.E95.D.829

Santosa, H., Hong, M. J., and Hong, K.-S. (2014). Lateralization of music process ing with noises in the auditory cortex: an fNIRS study. Front. Behav. Neurosci. 8:418. doi: 10.3389/fnbeh.2014.00418

Santosa, H., Hong, M. J., Kim, S.-P., and Hong, K.-S. (2013). Noise reduction in functional near-infrared spectroscopy signals by independent component analysis. Rev. Sci. Instrum. 84:073106. doi: 10.1063/1.4812785

Sardouie, S. H., and Shamsollahi, M. B. (2012). Selection of efficient features for discrimination of hand movements from MEG using a BCI competition IV data set. Front. Neurosci. 6:42. doi: 10.3389/fnins.2012.00042

Sato, H., Tanaka, N., Uchida, M., Hirabayashi, Y., Kanai, M., Ashida, T., et al. (2006). Wavelet analysis for detecting body-movement artifacts in optical topography signals. Neuroimage 33, 580-587. doi: 10.1016/j.neuroimage.2006. 06.028

Sato, H., Yahata, N., Funane, T., Takizawa, R., Katura, T., Atsumori, H., et al. (2013). A NIRS-fMRI investigation of prefrontal cortex activity during a working memory task. Neuroimage 83C, 158-173. doi: 10.1016/j.neuroimage.2013. 06.043

Schudlo, L. C., and Chau, T. (2014). Dynamic topographical pattern classification of multichannel prefrontal NIRS signals: II. Online differentiation of mental arithmetic and rest. J. Neural Eng. 11:016003. doi: 10.1088/17412560/11/1/016003
Seo, Y., Lee, S., Koh, D., and Kim, B.-M. (2012). Partial least squares-discriminant analysis for the prediction of hemodynamic changes using near-infrared spectroscopy. J. Opt. Soc. Korea 16, 57-62. doi: 10.3807/JOSK.2012.16.1.057

Shin, J., and Jeong, J. (2014). Multiclass classification of hemodynamic responses for performance improvement of functional near-infrared spectroscopy-based brain-computer interface. J. Biomed. Opt. 19:067009. doi: 10.1117/1.JBO.19.6.067009

Sitaram, R., Zhang, H. H., Guan, C. T., Thulasidas, M., Hoshi, Y., Ishikawa, A., et al. (2007). Temporal classification of multichannel near-infrared spectroscopy signals of motor imagery for developing a brain-computer interface. Neuroimage 34, 1416-1427. doi: 10.1016/j.neuroimage.2006.11.005

Soekadar, S. R., Witkowski, M., Cossio, E. G., Birbaumer, N., and Cohen, L. G. (2014). Learned EEG-based brain self-regulation of motor-related oscillations during application of transcranial electric brain stimulation: feasibility and limitations. Front. Behav. Neurosci. 8:93. doi: 10.3389/fnbeh.2014. 00093

Stangl, M., Bauernfeind, G., Kurzmann, J., Scerer, R., and Neuper, C. (2013). A hemodynamic brain-computer interface based on real-time classification of near infrared spectroscopy signals during motor imagery and mental arithmetic. J. Near Infrared Spectrosc. 21, 157-171. doi: 10.1255/ jnirs. 1048

Tachtsidis, I., Koh, P. H., Stubbs, C., and Elwell, C. E. (2010). "Functional optical topography analysis using statistical parametric mapping (SPM) methodology with and without physiological confounds," in Oxygen Transport to Tissue XXXI, eds E. Takahashi and D. F. Bruley (Boston, MA, Springer), 237-243

Tai, K., and Chau, T. (2009). Single-trial classification of NIRS signals during emotional induction tasks: towards a corporeal machine interface. J. Neuroeng. Rehabil. 6:39. doi: 10.1186/1743-0003-6-39

Takahashi, T., Takikawa, Y., Kawagoe, R., Shibuya, S., Iwano, T., and Kitazawa, S (2011). Influence of skin blood flow on near-infrared spectroscopy signals measured on the forehead during a verbal fluency task. Neuroimage 57, 991-1002. doi: 10.1016/j.neuroimage.2011.05.012

Tanaka, H., and Katura, T. (2011). Classification of change detection and change blindness from near-infrared spectroscopy signals. J. Biomed. Opt. 16:087001 doi: $10.1117 / 1.3606494$

Tomita, Y., Vialatte, F. B., Dreyfus, G., Mitsukura, Y., Bakardjian, H., and Cichocki, A. (2014). Bimodal BCI using simultaneously NIRS and EEG. IEEE Trans. Biomed. Eng. 61, 1274-1284. doi: 10.1109/TBME.2014.2300492

Turnip, A., and Hong, K.-S. (2012). Classifying mental activities from EEG-P300 signals using adaptive neural network. Int. J. Innovat. Comput. Inform. Control 8, 6429-6443

Turnip, A., Hong, K.-S., and Jeong, M.-Y. (2011). Real-time feature extraction of EEG-based P300 using adaptive nonlinear principal component analysis Biomed. Eng. Online 10:83. doi: 10.1186/1475-925X-10-83

Utsugi, K., Obata, A., Sato, H., Aoki, R., Maki, A., Koizumi, H., et al. (2008). GO-STOP control using optical brain-computer interface during calculation task. IEICE T. Commun. E91B, 2133-2141. doi: 10.1093/ietcom/e91-b. 7.2133

van der Heiden, L., Liberati, G., Sitaram, R., Kim, S., Jaœkowski, P., Raffone, A., et al. (2014). Insula and inferior frontal triangularis activations distinguish between conditioned brain responses using emotional sounds for basic BCI communication. Front. Behav. Neurosci. 8:247. doi: 10.3389/fnbeh.2014 00247

Villringer, A., and Chance, B. (1997). Non-invasive optical spectroscopy and imaging of human brain function. Trends Neurosci. 20, 435-442. doi: 10.1016/S01662236(97)01132-6

Villringer, A., Planck, J., Hock, C., Schleinkofer, L., and Dirnagl, U. (1993). Near infrared spectroscopy (NIRS): a new tool to study hemodynamic changes during activation of brain function in human adults. Neurosci. Lett. 154, 101-104. doi: 10.1016/0304-3940(93)90181-J

Wang, D., Miao, D., and Blohm, G. (2012). Multi-class motor imagery EEG decoding for brain-computer interfaces. Front. Neurosci. 6:151. doi: 10.3389/fnins.2012.00151

Weiskopf, N. (2012). Real-time fMRI and its application to neurofeedback. Neuroimage 62, 682-692. doi: 10.1016/j.neuroimage.2011.10.009

Weiskopf, N., Mathiak, K., Bock, S. W., Scharnowski, F., Veit, R., Grodd, W., et al. (2004). Principles of a brain-computer interface (BCI) based on real-time functional magnetic resonance imaging (fMRI). IEEE Trans. Biomed. Eng. 51, 966-970. doi: 10.1109/TBME.2004.827063 
Wolpaw, J. R., Birbaumer, N., McFarland, D. J., Pfurtscheller, G., and Vaughan, T. M. (2002). Brain-computer interfaces for communication and control. Clin. Neurophysiol. 113, 767-791. doi: 10.1016/S1388-2457(02)00057-3

Yoshino, K., and Kato, T. (2012). Vector-based phase classification of initial dips during word listening using near-infrared spectroscopy. Neuroreport 23, 947-951. doi: 10.1097/WNR.0b013e328359833b

Zhang, Q., Brown, E. N., and Strangman, G. E. (2007). Adaptive filtering to reduce global interference in evoked brain activity detection: a human subject case study. J. Biomed. Opt. 12:064009. doi: 10.1117/1.2804706

Zhang, Q., Strangman, G. E., and Ganis, G. (2009). Adaptive filtering to reduce global interference in non-invasive NIRS measures of brain activation: how well and when does it work?. Neuroimage 45, 788-794. doi: 10.1016/j.neuroimage.2008.12.048

Zhang, Y., Brooks, D., Franceschini, M., and Boas, D. (2005). Eigenvector-based spatial filtering for reduction of physiological interference in diffuse optical imaging. J. Biomed. Opt. 10:011014. doi: 10.1117/1.1852552

Zimmermann, R., Marchal-Crespo, L., Edelmann, J., Lambercy, O., Fluet, M.C., Riener, R., et al. (2013). Detection of motor execution using hybrid
fNIRS-biosignal BCI: a feasibility study. J. Neuroeng. Rehabil. 10:4. doi: 10.1186/1743-0003-10-4

Conflict of Interest Statement: The authors declare that the research was conducted in the absence of any commercial or financial relationships that could be construed as a potential conflict of interest.

Received: 27 October 2014; accepted: 02 January 2015; published online: 28 January 2015.

Citation: Naseer N and Hong K-S (2015) fNIRS-based brain-computer interfaces: $a$ review. Front. Hum. Neurosci. 9:3. doi: 10.3389/fnhum.2015.00003

This article was submitted to the journal Frontiers in Human Neuroscience. Copyright (c) 2015 Naseer and Hong. This is an open-access article distributed under the terms of the Creative Commons Attribution License (CC BY). The use, distribution or reproduction in other forums is permitted, provided the original author(s) or licensor are credited and that the original publication in this journal is cited, in accordance with accepted academic practice. No use, distribution or reproduction is permitted which does not comply with these terms. 